\title{
Factors Influencing the Uptake of HIV Counselling and Testing Services: The Case of the Employees of the Namibian Correctional Service at Elizabeth Nepemba Correctional Facility
}

\author{
Tangeni Velikoshi ${ }^{1}$, Burt Davis ${ }^{1} \&$ Daniel Opotamutale Ashipala ${ }^{2}$ \\ ${ }^{1}$ Africa Centre for HIV/AIDS Management, Faculty of Economic and Management Sciences, Stellenbosch \\ University (SU), Stellenbosch, South Africa \\ ${ }^{2}$ Department of General Nursing Science, School of Nursing, Faculty of Health Sciences, University of Namibia \\ (UNAM), Rundu, Namibia \\ Correspondence: Mr Tangeni Velikoshi, Deans of Students, University of Namibia (UNAM), Private Bag 13301, \\ 340 Mandume Ndemufayo Ave, Pionierspark, Windhoek, Namibia. Tel: 61-206-4987. E-mail: \\ tvelikoshi@unam.na
}

Received: September 9, 2018 Accepted: September 29, 2018 Online Published: October 24, 2018

doi:10.5539/gjhs.v10n11p179 URL: https://doi.org/10.5539/gjhs.v10n11p179

\begin{abstract}
Despite the growing public awareness about the burden of HIV and AIDS in Namibia, HIV Counselling and Testing (HCT) uptake remains to be low. The aim of this study was to explore factors influencing the HCT uptake amongst correctional officers deployed at Elizabeth Nepemba Correctional Facility (ENCF) in Rundu, Namibia. The study employed a quantitative approach, and a sample of 31 participants was constituted who completed self-administered questionnaires. This research focused on correctional officers deployed at ENCF. Participants were randomly selected from the employee list. It was found that the majority of the respondents (74\%) accessed HCT services in the past twelve months, of which $31 \%$ indicated having tested at Elizabeth Nepemba HCT Facility. Factors such as confidentiality and privacy issues, the condition of service and staff competency along with accessibility, fear of rejection from families and friends, information provision, education, future planning and risky behaviour were identified as having an influence on HCT uptake. This study concluded that there is a need for more awareness campaigns, information dissemination and involvement of stakeholders to address HIV-related issues for correctional officers at ENCF. This study recommends that the AIDS Committee and employee wellness department should spearhead workshops, information dissemination and educational programmes for correctional officers, so to improve their visibility and influence as well as improve HCT uptake. In addition, Regular workshops and seminars should be organized to empower correctional officers with the knowledge and skills related to HIV behavioural change; as well as resolve any issues related to fear of stigma or rejection.
\end{abstract}

Keywords: HIV Counselling and Testing, HCT, services, uptake, correctional facility

\section{Introduction}

HIV Counselling and Testing (HCT) is the process of providing counselling to an individual to enable him or her to make an informed choice about being tested for HIV (UNAIDS, 2016). Such a decision must be entirely the choice of an individual, and they must be assured of confidentiality. An individual's decision to test or not to test for HIV can be influenced by a number of factors both at the individual and service provider level. According to the National Department of Health South Africa (2016) people seek HCT if their partner or child dies, due to chronic or recurrent illnesses, planning of getting married or have a baby, to achieve a peace of mind and when they are worried about their partner's behaviour.

As an entry point for prevention and care, it is acknowledged that HCT is an effective strategy for both prevention and care. According to WHO (2017) Counselling and Testing (CT) is the primary access point to HIV and AIDS clinical care and psychological support and provides an opportunity for education and motivation to modify behavior aimed at reducing the risk of HIV infection. However, information and education alone have no transitory effect-eroded over time by contradictory pressures. In contrast, knowledge that leads to action, such as HIV and AIDS CT participation, promotes risk reduction through increasing perception of risk, self-efficacy and personal skill through the reinforcement of social norms and responsibilities (Miller, Moore, \& Peng, 2013). Hence, HCT is 
vital in all sectors including correctional settings. This study will investigate how HCT is utilized by correctional officers at Elizabeth Nepemba Correctional Facility (ENCF) and the factors affecting the utilization thereof.

According to MoHSS (2011), there are three approaches to conducting HCT: the client-initiated approach, provider-initiated approach and the home-based approach. Client Initiated Counselling and Testing (CICT) allow clients to seek out the service, while Provider Initiated Counselling and Testing (PICT) empowers the health care worker to recommend HIV testing to patients as part of routine health care services. Home-based HCT brings HCT services to the home. The PICT in health facilities and hospitals can improve diagnosis and save lives. Currently in Namibia, HIV testing is offered to patients in Antenatal clinics (ANC), maternity wards, medical and surgical wards, outpatient departments, sexual transmitted infection (STI) units, and Tuberculosis (TB) clinics, (MoHSS, 2016).

Data from HCT helps government -particularly those spearheading the health programs- to understand the prevalence, incidence and socio-demographic dimensions of health condition. Thus, HCT is not only useful in knowing your status but it helps in the development of policies, interventions programs and behavioural change campaign geared toward the reduction of HIV transmission (WHO, 2017). This situation poses major threats to the prevention efforts, including missed opportunities by many eligible Namibians to access treatment, care and support services. Namibia has one of the best developed Antiretroviral Therapy (ART) programs in SSA with free drugs available to $85 \%$ of those who are living positively and need them; but with many possible individuals not knowing their HIV status the therapy remains underutilized (MoHSS, 2013). Furthermore, low level of participation in routine HCT poses significant challenges to prevention, care and treatment programs. Firstly, individuals with high-risk behavioural profiles are at greater risk of contracting and passing on the virus if they are unaware of their or their partner's status. Secondly, delayed enrolment into HIV and AIDS care and treatment programs is associated with worse long-term health outcomes for infected persons. Also, infected persons who delay or avoid starting ART may, as they fall ill, be a greater financial burden on families and the health care system (MoHSS, 2016).

Just like any other population, HIV transmission is known to occur in correctional population in Namibia. The available data are of inmates' population which estimates a high HIV prevalence in correctional facilities in Namibia, (MoHSS, 2013). Correctional officers are among the core groups most at risk of HIV transmission and acquisition due to their occupation and lifestyle (MoHSS, 2013). It is essential to assess the knowledge, attitudes and perception of correctional officers as they are subjected to high mobility, location, health and other personal risks to themselves and their families, (UNAIDS \& USAIDS, 2017). As far as could be ascertained, there is no data available on the prevalence rate among correctional officers in the Namibian Correctional Service (NCS), in particular also not at ENCF, the focus of this research (NCF, 2012).

ENCF is equipped with a HCT facility aimed to cater for both correctional officers and the inmate population. Facility records indicate a low utility rate of HCT services amongst correctional officers in the past two years. Correctional officers are considered to be a high-risk group for HIV infection, the low uptake rate of HCT services is a cause for concern. To date, no study was done to identify factors that may be associated with the HCT uptake at ENCF. Therefore, identifying factors that may be associated with the observed low uptake of HCT in the correctional facility may make a valuable contribution to strengthening the national responses to HIV and AIDS epidemic in the correctional service context. This study will therefore aim to establish to what extent correctional officers utilize such services and what motivates or de-motivates them to do so.

Limited HIV and AIDS activities have been directed towards correctional officers at ENCF. A low uptake rate of HCT services by employees exists at ENCF, in addition to the prevalence rate among correctional officers in Namibia not being known. As mentioned, correctional officers are considered to be a high-risk group for HIV infection. The above phenomenon has led to the formulation of the research question: What are the factors contributing to the utilization of HCT amongst correctional officers at the ENCF?

\subsection{Goals and Objectives}

The goal of the study was to identify the factors influencing correctional officer's utilization of HCT services. Its objectives were to establish correctional officer's perception on HCT services and establish to what extent they utilize the HCT services.

\section{Research Design and Methods}

\subsection{Design}

A cross-sectional quantitative design utilizing a descriptive design was conducted (Van der Walt, Brink, \& Van Rensburg, 2018). This study employed a quantitative approach as a preferably, efficient and inexpensive method in 
collecting data from a large number of respondents in a survey (Grove, Burns, \& Gray, 2013).

\subsection{Study Population}

The study population included all Correctional Officers deployed at ENCF in Kavango East Region, Namibia.

\subsection{Inclusion and Exclusion Criteria}

All Correctional Officer deployed at ENCF who were randomly selected from the employee list and were willing to participate were included in the study. Correctional Officers who were not selected were excluded from the study. In addition, Correctional Officers who were not willing to participate were also excluded from the study.

\subsection{Sampling and Sample size}

Participants were randomly selected from the employee list of 172 members. Simple random sampling technique was used and every third officer on the list was selected for participation. A total number of 50 Correctional Officers were selected; fifty (50) data collection instruments were prepared and circulated to all selected Correctional Officers at ENCF.

\subsection{Data Collection Tool}

In this study, data was collected using questionnaires as primary source of data collection. This data collection method was used for the study, as it is considered to be a relevant tool to use when the researcher seeks to collect data from larger sample (Maree, 2016).

\subsection{Data Collection Methods}

The researcher distributed 50 questionnaires to participants, and a total number of 31 questionnaires were completed and returned.

\subsection{Data Analysis}

Data analysis from the questionnaires was presented as descriptive statistics and evaluated with quantitative, computerised statistical techniques, using SPSS version 24.

\subsection{Ethical Considerations}

HIV and AIDS are considered as sensitive issues due to the nature of the stigma and discrimination surrounding the disease. Participants in this study were assured confidentiality and anonymity. They were not obliged to divulge their names or personal particulars expect their gender, age and educational background. An informed consent form was considered prior participation. Participants were assured that research material and all documents with their response are going to be kept safe in an area only accessible to the researcher. 


\section{Results}

\subsection{Socio Demographic Description of Study Participants}

3.1.1 Gender of the Participants

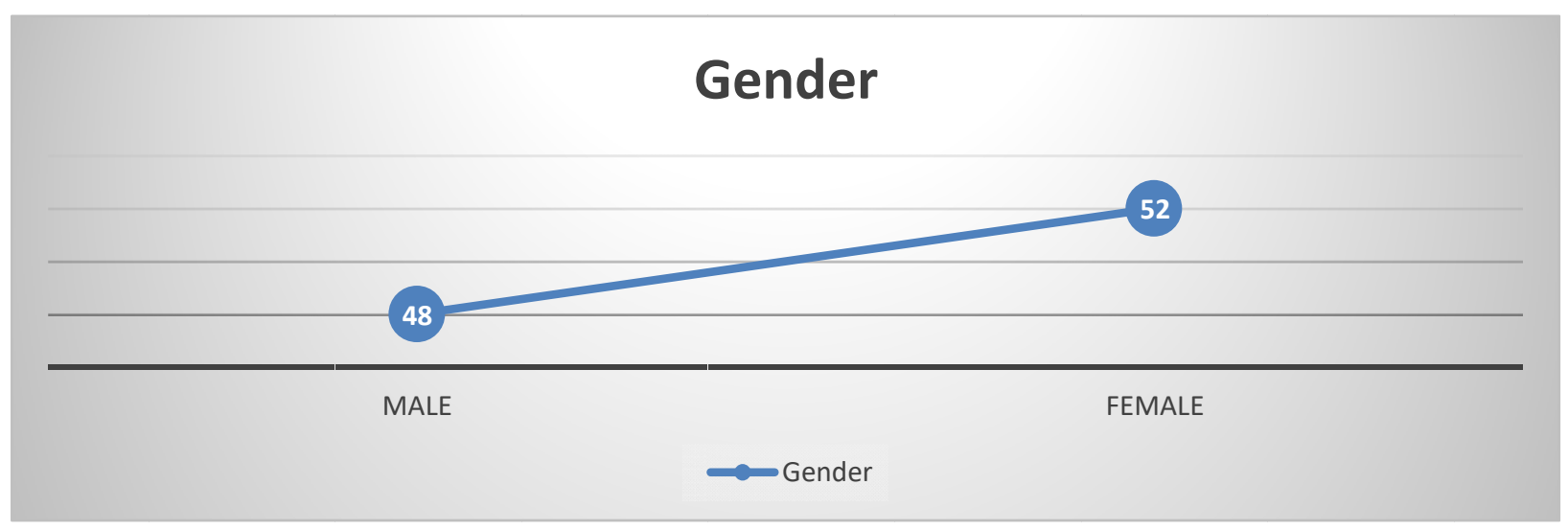

Figure 1. Gender distribution of participants

Figure 1 outlines the gender ratio of males and females who took part in the study. It indicates that more males compared to females participated as revealed by $52 \%$ (16) against $48 \%$ (15) females.

\subsubsection{Age Distribution of Participants}

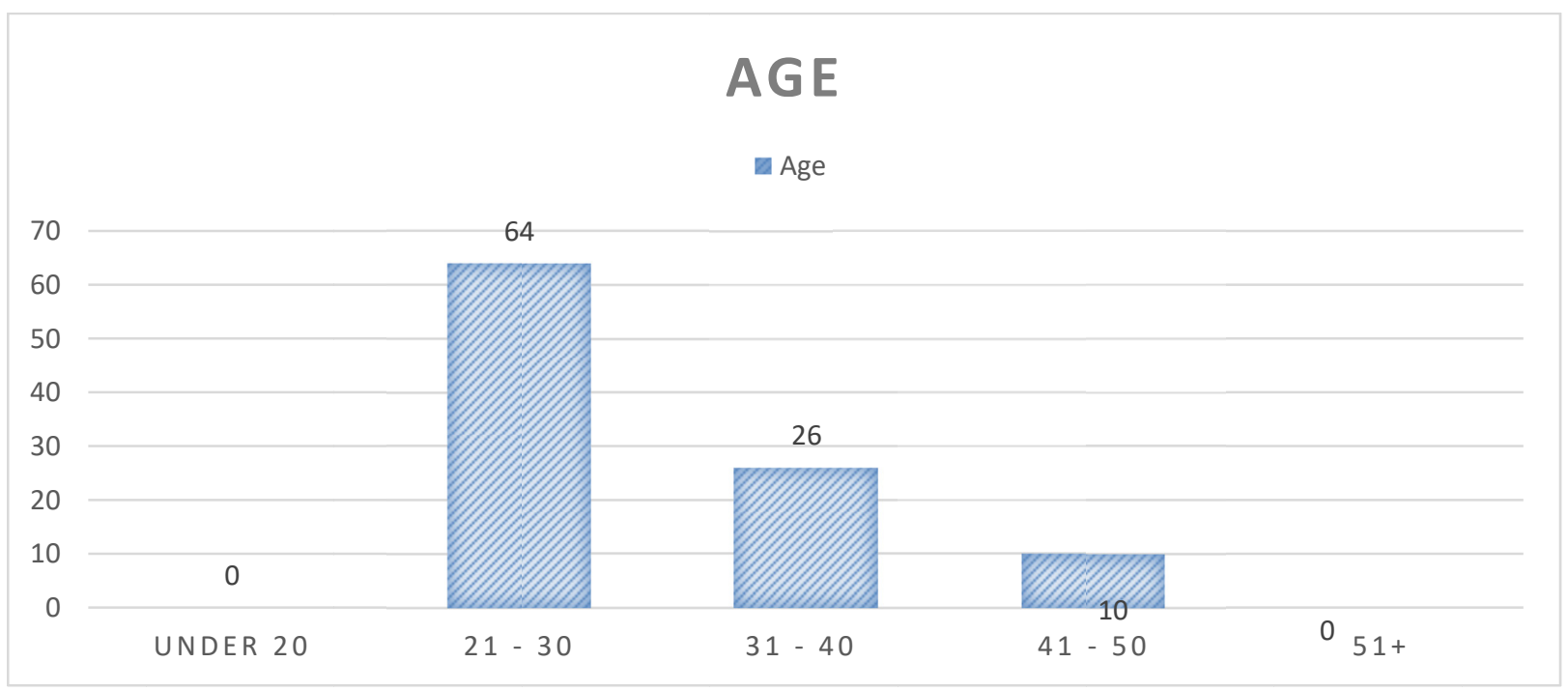

Figure 2. Age distribution of participants.

Figure 2 outlines the distribution of respondents by age. There were no participants under the age of 20 and above the age of 51 . The majority of respondents (in total 20 ) were between the age of $21-30$, with $26 \%$ in the age range of $31-40$ and only $10 \%$ in the range of $41-50$. 


\subsubsection{Marital status of participants}

\section{MARITAL STATUS}

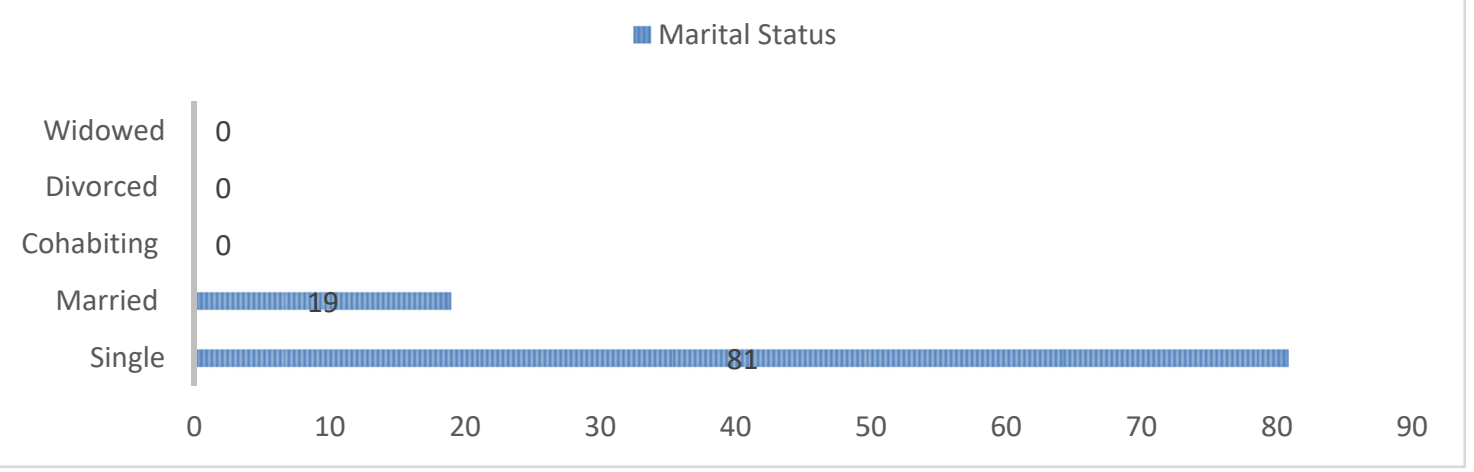

Figure 3. Distribution of Correctional Officers by marital status

Figure 3 indicates the marital distribution of respondents in the study. None of the respondents fell in the categories of cohabiting, divorced or widowed. Most of the respondents were single (81\%) while six respondents were married (19\%).

\subsubsection{Educational Background of Participants}

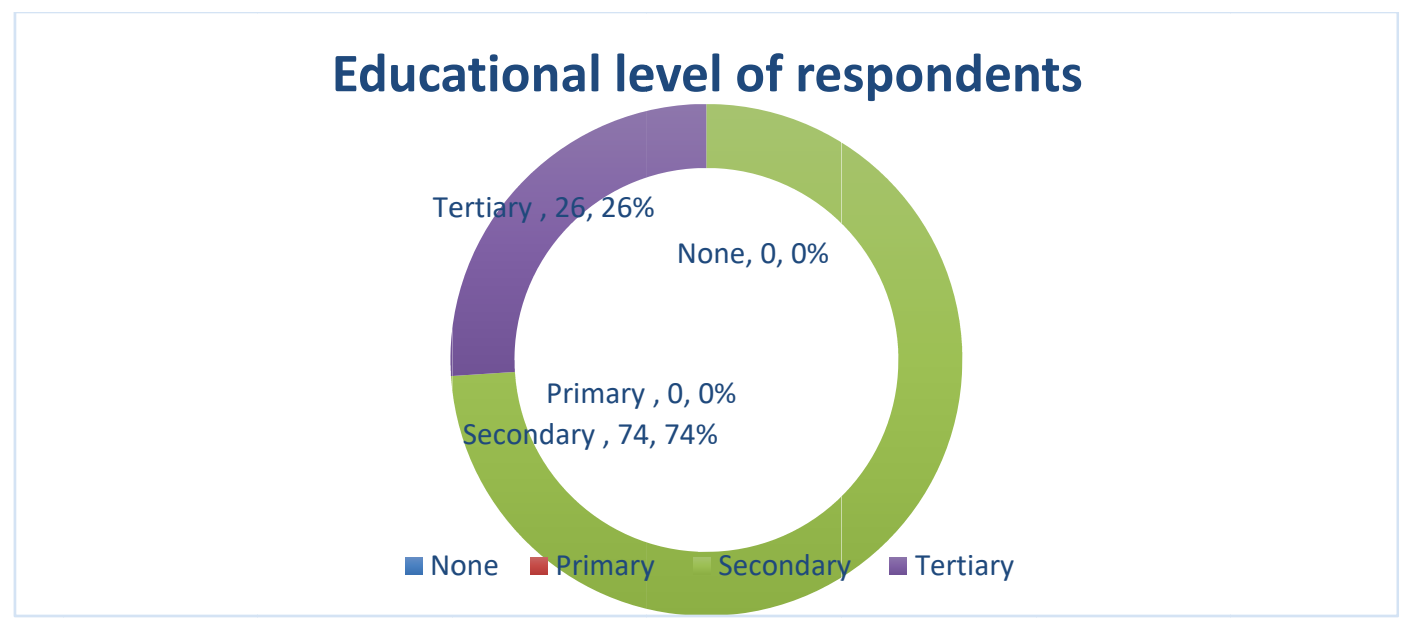

Figure 4. Distribution of participants according to educational level

Figure 4 indicates that the majority of respondents have secondary educational qualification. This translates to $74 \%$ of the total respondents. Trailing is eight respondents with post-matric qualification that represent $26 \%$. None of the respondents were without educational qualification or with primary educational level. 


\subsubsection{Distribution in Terms of Testing Reasons}

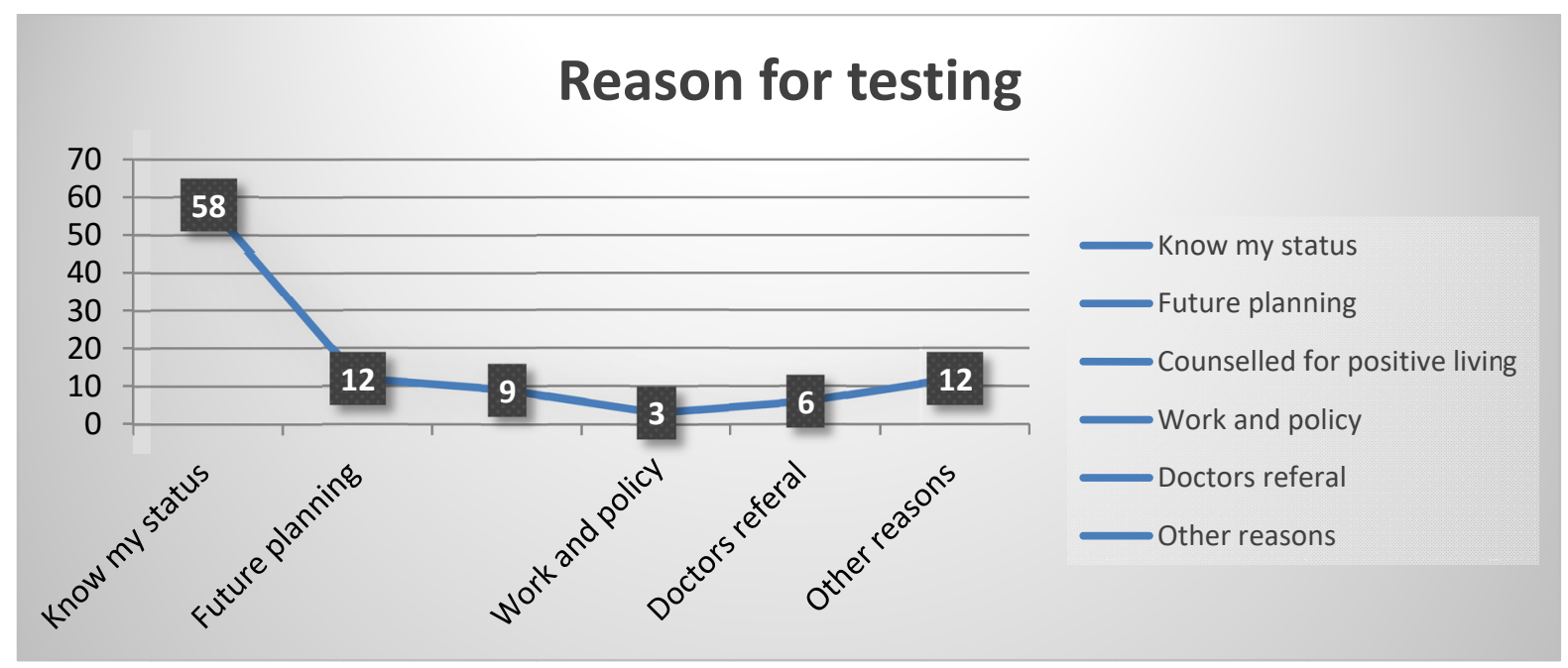

Figure 5. Distribution of participants in terms of testing reasons.

When asked about reasons they went for HCT, the majority of the respondents (19 in total) indicated that they went for an HIV test just to know their status, which translate to $58 \%$. Twelve percent of the respondents went due to future planning and other reasons, $9 \%$ due to counselling and positive living reasons and $6 \%$ were referred by the doctors. Only $3 \%$ of the respondents were tested because of work and policy reasons.

\subsubsection{Participants Reasons for Selecting A Testing Facility}

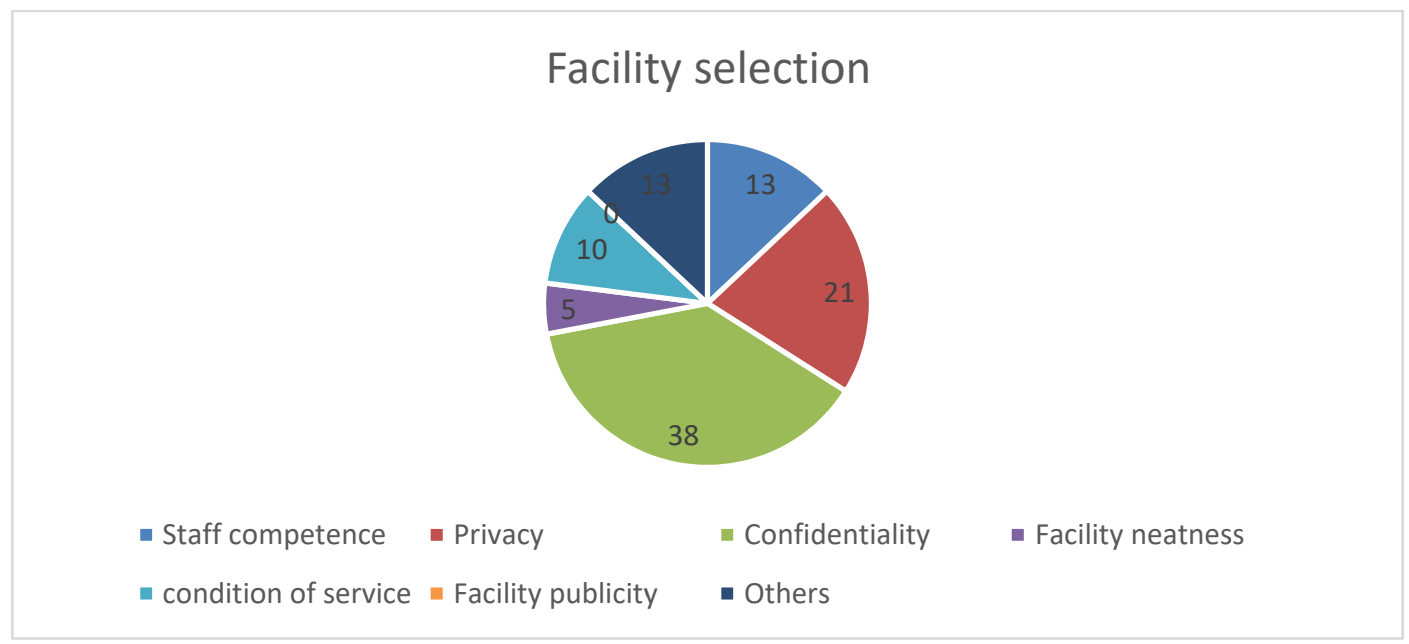

Figure 6. Distribution of participants by facility selection.

In response to the reason(s) why they selected a particular HCT facility for HIV testing, the majority of respondents by $38 \%$ chose a facility due to confidentiality. Trailing behind is the eight respondents because of privacy, representing $21 \%$. Five respondents chose because of staff competence and other five indicated other reasons, which constitute $13 \%$ each. Ten percent of respondents selected a facility due to condition of service and only $5 \%$ did their selection based on facility neatness (i.e. two participants out of the total sample).

\subsection{Validity and Liability}

The sample size was sufficiently large and was drawn from the large group of the same characteristic to the sample. The questionnaire was pre - tested prior to actual data collection with similar characteristic to the 
sample. The questionnaire was administered in language understood by both respondents and the researchers. Data quality checks were done in the field as well as before and data processing to ensure completeness and consistency. To address reliability in this study, the same questionnaire was used to collect data from all the participants and this was only done by the researcher alone.

\section{Discussion}

The demographic characteristics of the study sample indicate that there were more males who took part in the study. This could be as a result of more male compared to female correctional officers at ENCF, or simply that males were more willing to participate in this study. The study found that the majority of correctional officers who participated in the study were in the age range of $21-40$. Furthermore, the majority of respondents have secondary education. It was cited that being older youth and having an educational level of secondary and above are factors having a positive correlation with willingness to test for HIV. A similar conclusion could therefore possibly be drawn for this study, as most correctional officers can be categorized in a younger cohort and have secondary education, which is the category associated with high testing. This was indeed the case, as the study found that $74 \%$ of participants tested for HIV in the last 12 months at private, Elizabeth Nepemba and public health facilities. With regard to correctional officers rank ration, it was noted that more junior officers took part in the study than senior officers. This can be due to the fact that there are few senior officers at the correctional facility.

There are several reasons that persuaded individuals to seek HCT services and their decision where to utilize such services. In the case of correctional officers as derived from this study, their main desire to seek HCT services was to simply know their status; while treating information as confidential had the biggest influence on respondents' decision to select a particular facility to utilize the said services.

The study found that $81 \%$ of correctional officer's decision to test for HIV would not be influenced by the accessibility of the testing facility. This could be because ENCF is equipped with a HIV testing facility; as well as the relatively easy access to other public and private testing sites within the vicinity of Rundu. Most correctional officers would not to test for HIV because they fear rejection from family and friends. Thus, the fear of rejection from family and friends could be considered as a barrier to HCT services utilization. The possibility of losing their jobs was not a factor deterring them from testing. This may indicate a high level of job security among respondents.

The majority of correctional officers agreed that knowing that one's result will be kept confidential would motivate them to test for HIV. In addition, the majority of the respondents agreed that the skills, level of professionalism and attitudes of health workers will have an influence on whether they will test or not. This is in line with the findings of Arrey, Bilsen, Lacor, \& Deschepper (2015), who report that people are sometimes anxious about HIV testing if they fear there are confidentiality issues, while if it is not the case they would be more inclined to test.

In response to the questions on role models and motivation: Results showed that most respondents agreed that they would test for HIV if they were motivated by those they look up to testing. Equally, the majority of correctional officers agreed that if they have health concerns they might be positively influenced to go for HIV testing. Results further found that the majority of correctional officers agreed that they would test for HIV if they believe they are at the risk of contracting HIV. This is in line with quoted studies where it was cited that past exposure have shown an effect on HCT uptake (Stephenson, White, Darbes, Hoff, \& Sullivan, 2015).

The majority of respondents agreed that peer's support, reliability of the testing instrument and information provision on positive living would have a positive influence in their testing behaviour. This is in line with the literature, which indicated that clients should be helped to e.g. understand how antibody tests work and be provided with the necessary information so to make 5896 informed decisions (e.g. weighing the pros and cons of HIV testing). Access to information prepares individuals for difficulties they may face in future (Dapaah \& Senah, 2016). The majority of the general comments by correctional officer in this study were centered on education, information provision, confidentiality, and awareness campaigns to influence officers to test for HIV. It was noted to note that most correctional officer prefer including HIV information sharing during the monthly meeting as well as a conducive time and facility for discussions. They also felt the need for more workshops and called for the invitation of expertise to address the on HIV and AIDS related matters. Furthermore, they also suggested the establishment of support groups for correctional officers who test HIV positive and for those living with the virus. 
It was also found that some correctional officers seemed not to be aware of the existence of the facility's AIDS committee. This was revealed by their constant request of the formulating a committee that will be mandated with HIV and AIDS related affairs. Together with those that are aware of its existence, the correctional officers believed many of them will be better motivated to test if the communication and testing was done in their vernacular.

\section{Conclusions}

In terms of HCT, $74 \%$ of participants reported accessing HCT services in the last twelve months. The study revealed a number of factors that influence the HCT uptake of correctional officers, ranging from confidentiality issues, and privacy issues to the condition of service and staff competency. It further identified factors such as facility accessibility, fear of rejection from family and friends, information provision and education, future planning and risky behaviour as factors which can influence HCT uptake.

The study also showed that there is a need for awareness campaigns, information dissemination and involvement of stakeholders in order to enhance behavioural change and address health and high risk issues for correctional officers. In particular, more awareness around the functioning of the existing AIDS Committee should be done and the establishment of support mechanisms for HIV-related issues should be established. The study also revealed that more needs to be done to address the apparent fear of rejection from family and friends if they would test positive that respondents seem to display.

\subsection{Recommendations}

Based on the study findings, the following recommendations are made:

- The AIDS Committee should spearhead workshops, information dissemination and educational programmes for correctional officers, so to improve their visibility and influence as well as improve HCT uptake. The facility should involve stakeholders both at senior and junior level. It is necessary that correctional officers should be informed of the existence of the intensified awareness programs.

- Regular workshops and seminars should be organized to empower correctional officers with the knowledge and skills related to HIV behavioural change; as well as resolve any issues related to fear of stigma or rejection by family members because of their HIV-status.

- The study revealed that correctional officers are influenced by the competencies of health workers. Regular training or refresher courses for health workers are recommended. In achieving this goal, additional health workers may need to be employed.

- Facility leadership also needs to take more initiative or ownership and find ways to motivate employees to seek HCT. From the study findings, if they do take the initiative, motivating their members (i.e. those that look up to them) could be a successful way to increase HCT uptake.

- A comparative study on a larger scale needs to be conducted in other correctional facilities explore similar influences in those facilities so to draw comparisons to the current study.

\subsection{Study Delimitations and Limitation}

The study was limited to the Correctional Officers deployed at ENCF and inmates were excluded. Moreover, and given the sensitive nature of the study, the participants may have given limited information which may have influenced the findings. As discussed, few studies were done on HCT uptake amongst correctional officers both in Namibia and on the African continent.

\section{Acknowledgements}

We thank all the respondents who took time to participate in this study; without their participation this study would not have been possible. We also thank the Ministry of Prisons and Correctional Services for having granted us permission to conduct research in its correctional facility.

\section{Competing Interest Statement}

The authors declare that there is no conflict of interest.

\section{References}

Arrey, A. E., Bilsen, J, Lacor, P., \& Deschepper, R. (2015). "It is my secret": Fear of disclosure among Sub-Saharan African migrant women living with HIV/AIDS in Belgium. PLoS ONE, 10(3), E0119653. https://doi.org/10.1371/journal.pone.0119653

Dapaah, M. J., \& Senah, A. K. (2016). HIV/AIDS clients, privacy and confidentiality; the case of two health 
centres in the Ashanti Region of Ghana. BMC medical ethics, $17,41$. https://doi.org/10.1186/s12910-016-0123-3

Grove, S. K., Burns, S., \& Gray, J. R. (2013). The practice of nursing research: Appraisal, synthesis, and generation of evidence (7th ed.). St Louis: Elsevier Saunders.

Maree, K. (2016). First step in research. Pretoria: Van Schaik.

Miller, E. R., Moore, J. M., \& Peng, B. (2013). Harm reduction behind bars: Prison workers perspectives. SAGE, 2013, 1-12. https://doi.org/10.1177/2158244013494209

MoHSS. (2011). National Guidelines for HIV Counselling and Testing. Windhoek: Ministry of Health and Social Services.

MoHSS. (2013). Demographic and Hemalth Survey 2013. Windhoek, Namibia: Ministry of Health and Social Services.

MoHSS. (2016). National Guide for Antiretroviral Therapy (5th ed.). Windhoek, Namibia: Ministry of Health and Social Services.

MoHSS. (2016). Surveillance Report of the 2016 National HIV Sentinel Survey. Windhoek, Namiba: Ministry of Health and Social Services.

Namibian Correctional Service [NCF]. (2012). Correctional Journal Namibian Correctional Service. (2011). Elizabeth Nepemba Inaguration Booklet. Windhoek: NCS.

National Department of Health South Africa. (2016). Disclosure guidelines for children and adolescents in the context of HIV, TB and non-communicable diseases. Pretoria: National Department of Health South Africa.

Stephenson, R., White, D., Darbes, L., Hoff, C., \& Sullivan, P. (2015). HIV Testing and Perceptions of Risks of HIV infection among MSM with the main partners. PMC, 19(3), 553-560. https://doi.org/10.1007/s10461-014-0862-4

UNAIDS and USAIDS. (2017). AIDS and the Military: UNAIDS Point of view. AIDS \& man who have sex with men: UNAIDS campaign. Geneva: UNAIDS.

UNAIDS. (2016). WHO guidelines on HIV infection and AIDS in prison. Switzerland: UNAIDS.

Van der Walt, C., Brink, H., \& Van Rensburg, G. (2018). Fundamentals of research methodology for health care professionals (4th ed.). Cape Town: Juta.

World Health Organisation [WHO]. (2017). HIV testing services: new opportunities and ongoing challenges. Switzerland, Geneva: World Health Organisation.

\section{Copyrights}

Copyright for this article is retained by the author(s), with first publication rights granted to the journal.

This is an open-access article distributed under the terms and conditions of the Creative Commons Attribution license (http://creativecommons.org/licenses/by/4.0/). 